PROCEEDINGS OF THE

AMERICAN MATHEMATICAL SOCIETY

Volume 131, Number 10, Pages 3155-3161

S 0002-9939(03)07048-5

Article electronically published on April 1, 2003

\title{
AN UNCERTAINTY PRINCIPLE ON HOMOGENEOUS TREES
}

\author{
FRANCESCA ASTENGO
}

(Communicated by Andreas Seeger)

\begin{abstract}
Let $\mathfrak{X}$ be a homogeneous tree of degree $q+1$. We prove an uncertainty principle in this setting regarding "exponentially decreasing" functions on trees whose Fourier transforms have a "deep zero".
\end{abstract}

\section{INTRODUCTION}

The classical Heisenberg Uncertainty Principle may be phrased in the language of Fourier transforms by saying roughly that both $f$ and $\widehat{f}$ cannot be well localized unless $f=0$.

Various forms of the Uncertainty Principle in other settings have attracted attention in the past few years. Many references can be found in the survey by G. B. Folland and A. Sitaram [7] or in the book by V. Havin and B. Jöricke [9]. Recently, many authors have discovered forms of this principle on Lie groups, such as the $n$-dimensional motion group, nilpotent Lie groups, noncompact semisimple Lie groups, and symmetric spaces (see the references in [7], [8] and in [10]).

In this paper we consider the case of a homogeneous tree of degree $q+1$, i.e., a connected graph with no loops, in which every vertex is adjacent to $q+1$ other vertices. A homogeneous tree may be viewed as a discrete model of the hyperbolic space, and many authors [6], [3] have pointed out the analogy of harmonic analysis on these structures. In this line of research, it is natural to study uncertainty principles on homogeneous trees.

When $q=1$, the underlying group is the integers, and a form of the Uncertainty Principle is Volberg's First Theorem [9], which can be stated as follows. Let $\alpha>0$ and suppose $f$ is a nontrivial function on $\mathbb{Z}$ such that $f(n)=O\left(e^{-\alpha|n|}\right)$ as $n \rightarrow-\infty$. Moreover suppose that its Fourier transform $\widehat{f}$ is integrable on the torus $\mathbb{T}$. Then $\int_{\mathbb{T}} \log |\widehat{f}| d x>-\infty$.

Our result is Theorem [2.1, where we extend this kind of Uncertainty Principle to the case $q>1$. In the spirit of [1, it is stated in terms of the Helgason-Fourier transform. Proposition 2.2 shows that our result is sharp.

It is a pleasure to thank M. Cowling and G. Mauceri for their valuable help.

Received by the editors May 6, 2002.

2000 Mathematics Subject Classification. Primary 43A85; Secondary 22E35.

(C)2003 American Mathematical Society 


\section{Homogeneous tReES}

Let $\mathfrak{X}$ be a homogeneous tree of degree $q+1$. Details of the harmonic analysis on $\mathfrak{X}$ can be found in [5], [6]; here we mainly follow more recent work of M. G. Cowling, S. Meda and A. G. Setti [3].

We denote by $d$ the natural distance on $\mathfrak{X}, d(x, y)$ being the number of edges between the vertices $x$ and $y$. Let $o$ be a fixed reference point on $\mathfrak{X}$, and write $|x|$ for $d(x, o)$. We say that a function $f$ on $\mathfrak{X}$ is radial if $f(x)$ depends only on $|x|$.

Let $G$ be the group of automorphisms of the tree, i.e., of isometries of $(\mathfrak{X}, d)$, and let $K$ be the isotropy subgroup of $o$. Then $K$ is maximal compact in $G$, the tree $\mathfrak{X}$ may be canonically identified with the coset space $G / K$, and functions and radial functions on $\mathfrak{X}$ may be identified with $K$-right-invariant and $K$-bi-invariant functions on $G$, respectively.

A geodesic ray $\gamma$ in $\mathfrak{X}$ is a sequence $\left(\gamma_{n}\right)_{n \in \mathbb{N}}$ of points of $\mathfrak{X}$ such that $d\left(\gamma_{i}, \gamma_{j}\right)=$ $|i-j|$ for every $i, j \in \mathbb{N}$. We say that a vertex $x$ lies on $\gamma$ if there exists $n$ in $\mathbb{N}$ such that $x=\gamma_{n}$. Let $\gamma$ and $\gamma^{\prime}$ be geodesic rays; we say that $\gamma$ and $\gamma^{\prime}$ are equivalent if there exist integers $n_{0}, n_{1}$ such that $\gamma_{n}=\gamma_{n+n_{1}}^{\prime}$ for every $n \geq n_{0}$.

The boundary of $\mathfrak{X}$ is the set of equivalence classes of all geodesic rays and will be denoted by $\Omega$. It can also be realized as the set of all geodesic rays starting at the reference point $o$, since in every equivalence class there is such a ray.

Let $\gamma$ and $\gamma^{\prime}$ be equivalent geodesic rays and $g$ in $G$. Then it is easy to prove that $g \cdot \gamma$ and $g \cdot \gamma^{\prime}$ are equivalent. Hence the group $G$ acts also on the boundary $\Omega$. One can prove that $K$ acts transitively on the boundary; therefore there is a unique probability measure $\sigma$ on $\Omega$, which is also $G$-quasi-invariant. The Poisson kernel $P$ is defined to be the Radon-Nikodym derivative $d \sigma\left(g^{-1} \cdot \omega\right) / d \sigma(\omega)$. Since $\sigma$ is $K$ invariant,

$$
P(g k, \omega)=P(g, \omega) \quad \forall g \in G, \quad \forall k \in K, \quad \forall \omega \in \Omega,
$$

so the Poisson kernel $P$ can be regarded as a function on $\mathfrak{X} \times \Omega$.

Let $L$ be the Laplace operator on the tree, i.e., for any function $f$ on $\mathfrak{X}$,

$$
L f(x)=f(x)-\frac{1}{q+1} \sum_{y: d(x, y)=1} f(y) \quad \forall x \in \mathfrak{X} .
$$

Then [5] chapter 2] for every fixed $\omega$ in the boundary, $P^{\frac{1}{2}+i z}(\cdot, \omega)$ is an eigenfunction of $L$ with eigenvalue $1-\eta(z)$, where $\eta(z)=\frac{2 q^{1 / 2}}{q+1} \cos (z \log q)$. The radial eigenfunction $\phi_{z}$ of the Laplace operator corresponding to the eigenvalue $1-\eta(z)$, normalized by the condition $\phi_{z}(o)=1$, is called a spherical function. It can be represented by [5, Proposition 2.4]

$$
\phi_{z}(x)=\int_{\Omega} P^{\frac{1}{2}+i z}(x, \omega) d \sigma(\omega) \quad \forall z \in \mathbb{C}, \quad x \in \mathfrak{X} .
$$

Given a smooth finitely supported function $f$ on the tree $\mathfrak{X}$, we define its HelgasonFourier transform $\mathcal{H} f: \Omega \times \mathbb{C} \rightarrow \mathbb{C}$ by

$$
\mathcal{H} f(\omega, z)=\sum_{x \in \mathfrak{X}} f(x) P^{\frac{1}{2}+i z}(x, \omega) \quad \forall(\omega, z) \in \Omega \times \mathbb{C} .
$$

If $f$ is radial, then $\mathcal{H} f$ does not depend on $\omega$ and

$$
\mathcal{H} f(\omega, z)=\sum_{x \in \mathfrak{X}} f(x) \phi_{z}(x) \quad \forall(\omega, z) \in \Omega \times \mathbb{C} .
$$


The definition of the Helgason-Fourier transform can be extended to more general classes of functions if one lets $z$ vary in a proper strip of the complex plane.

Let $\tau$ be the number $2 \pi / \log q$ and denote by $\mathbb{T}$ the torus $\mathbb{R} / \tau \mathbb{Z}$; we shall usually identify $\mathbb{T}$ with the interval $[-\tau / 2, \tau / 2)$ equipped with Haar measure $\frac{d s}{\tau}, d s$ being Lebesgue measure. With these conventions, the usual Fourier transform $\mathcal{F}$ on $\mathbb{Z}$ and its inverse read

$$
\mathcal{F} F(z)=\sum_{n \in \mathbb{Z}} F(n) q^{i n z}, \quad F(n)=\int_{-\tau / 2}^{\tau / 2} \mathcal{F} F(s) q^{-i s n} \frac{d s}{\tau} \quad \forall n \in \mathbb{Z}, \quad \forall z \in \mathbb{C},
$$

for suitable functions $F$ on $\mathbb{Z}$.

Define the meromorphic function $\mathbf{c}$ by the rule

$$
\mathbf{c}(z)=\frac{q^{1 / 2}}{q+1} \frac{q^{\frac{1}{2}+i z}-q^{-\frac{1}{2}-i z}}{q^{i z}-q^{-i z}} \quad \forall z \in \mathbb{C} \backslash(\tau / 2) \mathbb{Z} .
$$

Then [3] the following inversion formula holds:

$$
f(x)=c_{q} \int_{-\tau / 2}^{\tau / 2}|\mathbf{c}(s)|^{-2} \int_{\Omega} P^{\frac{1}{2}-i s}(x, \omega) \mathcal{H} f(\omega, s) d \sigma(\omega) \frac{d s}{\tau} \quad \forall x \in \mathfrak{X},
$$

where $c_{q}=\frac{q}{2(q+1)}$ for any $f$ such that

$$
\sup _{x \in \mathcal{X}}(1+|x|)^{m} q^{|x| / 2}|f(x)|<\infty \quad \forall m \in \mathbb{N} .
$$

The Helgason-Fourier transform can be factored as follows. Given a geodesic ray $\gamma$, we define the height function by

$$
h_{\gamma}(x)=\lim _{m \rightarrow \infty}\left(m-d\left(x, \gamma_{m}\right)\right)
$$

and we define the horocycle $\mathfrak{H}_{\gamma, h}$ with direction $\gamma$ and level $h$ in $\mathbb{Z}$ by

$$
\mathfrak{H}_{\gamma, h}=\left\{x \in \mathfrak{X}: h_{\gamma}(x)=h\right\} .
$$

Then $\mathfrak{X}$ decomposes into the disjoint union $\mathfrak{X}=\bigcup_{h \in \mathbb{Z}} \mathfrak{H}_{\gamma, h}$. Note that equivalent geodesic rays determine the same decomposition of $\mathfrak{X}$ into horocycles, hence the set of horocycles is in one-to-one correspondence with $\Omega \times \mathbb{Z}$.

Given a smooth finitely supported function $f$ on the tree $\mathfrak{X}$, we define its Abel transform $\mathcal{A f}: \Omega \times \mathbb{Z} \rightarrow \mathbb{C}$ by

$$
\mathcal{A} f(\omega, h)=q^{h / 2} \sum_{x \in \mathfrak{H}(\omega, h)} f(x) \quad \forall(\omega, h) \in \Omega \times \mathbb{Z} .
$$

Then

$$
\mathcal{H} f(\omega, z)=(\mathcal{F} \circ \mathcal{A}) f(\omega, z) .
$$

W. Betori and M. Pagliacci [2] proved that, if $f$ is a radial function on $\mathfrak{X}$, then

$$
\mathcal{A} f(\omega, h)=q^{|h| / 2}\left(f(|h|)+\frac{q-1}{q} \sum_{j \in \mathbb{Z}^{+}} f(|h|+2 j)\right) \quad \forall(\omega, h) \in \Omega \times \mathbb{Z} .
$$




\section{RESULTS}

We now state and prove our uncertainty principle.

Theorem 2.1. Let $\mathfrak{X}$ be a homogeneous tree of degree $q+1$ and let $\alpha>\frac{1}{2} \log q$. Suppose $f$ is a (measurable) function on $\mathfrak{X}$ such that

$$
|f(x)| \leq C e^{-\alpha|x|} \quad \forall x \in \mathfrak{X} ;
$$

moreover suppose that its Helgason-Fourier transform $\mathcal{H} f$ satisfies

$$
s \in \mathbb{T} \mapsto\|\mathcal{H} f(\cdot, s)\|_{L^{p}(\Omega)} \in L^{1}(\mathbb{T}) \quad \text { and } \quad \int_{\mathbb{T}} \log \|\mathcal{H} f(\cdot, s)\|_{L^{p}(\Omega)} \frac{d s}{\tau}=-\infty .
$$

Then $f=0$.

The proof shows that one can replace $L^{p}$ norms on $\Omega$ with other norms, such as dual norms.

Note that the hypotheses on the Helgason-Fourier transform side are fulfilled if there exists $s_{0}$ in $\mathbb{T}$ such that the derivatives of any order of the function $s \mapsto$ $\|\mathcal{H} f(\cdot, s)\|_{L^{p}(\Omega)}$ vanish at $s_{0}$. As in the case of $\mathbb{Z}[9$, chapters 2 and 3], the decay condition on the function $f$ may be slightly relaxed.

Proof. Let $\varphi$ be a smooth compactly supported function on $\Omega$ and consider the function $F_{\varphi}$ on $\mathbb{Z}$ defined by

$$
F_{\varphi}(h)=\int_{\Omega} \varphi(\omega) \mathcal{A} f(\omega, h) d \sigma(\omega) \quad \forall h \in \mathbb{Z} .
$$

Since $x \mapsto e^{-\alpha|x|}$ is a radial function on the tree, we have by equation (1.1)

$$
\begin{aligned}
\left|F_{\varphi}(h)\right| & \leq \int_{\Omega}|\varphi(\omega)|\left(q^{h / 2} \sum_{x \in \mathfrak{H}(\omega, h)}|f(x)|\right) d \sigma(\omega) \\
& \leq C \int_{\Omega}|\varphi(\omega)|\left(q^{h / 2} \sum_{x \in \mathfrak{H}(\omega, h)} e^{-\alpha|x|}\right) d \sigma(\omega) \\
& =C \int_{\Omega}|\varphi(\omega)| d \sigma(\omega)\left(q^{|h| / 2} e^{-\alpha|h|}+\frac{q-1}{q} \sum_{j=1}^{\infty} q^{j} e^{-\alpha(|h|+2 j)}\right) \\
& =C\|\varphi\|_{L^{1}(\Omega)}\left(e^{-\left(\alpha-\frac{1}{2} \log q\right)|h|}+\frac{(q-1) e^{-2 \alpha}}{1-q e^{-2 \alpha}} e^{-\alpha|h|}\right) \\
& \leq C_{q, \alpha, \varphi} e^{-\beta|h|}
\end{aligned}
$$

for some positive constants $C_{q, \alpha, \varphi}$ and $\beta$.

By Fubini's Theorem we obtain for every $s$ in $\mathbb{T}$

$$
\begin{aligned}
\left(\mathcal{F} F_{\varphi}\right)(s) & =\sum_{h \in \mathbb{Z}} F_{\varphi}(h) q^{i h s}=\sum_{h \in \mathbb{Z}} \int_{\Omega} \varphi(\omega) \mathcal{A} f(\omega, h) d \sigma(\omega) q^{i h s} \\
& =\int_{\Omega} \varphi(\omega) \mathcal{F} \circ \mathcal{A} f(\omega, s) d \sigma(\omega) \\
& =\int_{\Omega} \varphi(\omega) \mathcal{H} f(\omega, s) d \sigma(\omega) .
\end{aligned}
$$


Hence

$$
\begin{aligned}
\int_{\mathbb{T}}\left|\mathcal{F} F_{\varphi}(s)\right| \frac{d s}{\tau} & =\int_{\mathbb{T}}\left|\int_{\Omega} \varphi(\omega) \mathcal{H} f(\omega, s) d \sigma(\omega)\right| \frac{d s}{\tau} \\
& \leq\|\varphi\|_{L^{p^{\prime}}(\Omega)} \int_{\mathbb{T}}\|\mathcal{H} f(\cdot, s)\|_{L^{p}(\Omega)} \frac{d s}{\tau}<\infty
\end{aligned}
$$

i.e., the function $s \mapsto \mathcal{F} F_{\varphi}(s)$ is in $L^{1}(\mathbb{T})$. Moreover

$$
\begin{aligned}
\int_{\mathbb{T}} \log \left|\mathcal{F} F_{\varphi}(s)\right| \frac{d s}{\tau} & =\int_{\mathbb{T}} \log \left|\int_{\Omega} \varphi(\omega) \mathcal{H} f(\omega, s) d \sigma(\omega)\right| \frac{d s}{\tau} \\
& \leq \int_{\mathbb{T}} \log \left(\|\varphi\|_{L^{p^{\prime}}(\Omega)}\|\mathcal{H} f(\cdot, s)\|_{L^{p}(\Omega)}\right) \frac{d s}{\tau} \\
& =\log \left(\|\varphi\|_{L^{p^{\prime}}(\Omega)}\right)+\int_{\mathbb{T}} \log \left(\|\mathcal{H} f(\cdot, s)\|_{L^{p}(\Omega)}\right) \frac{d s}{\tau}=-\infty
\end{aligned}
$$

By the Uncertainty Principle on $\mathbb{T}$ and $\mathbb{Z}$ [9, First Volberg Theorem, p. 222], we conclude that $F_{\varphi}=0$. The function $\varphi$ being arbitrary, we infer that $\mathcal{A} f=0$, hence $f=0$.

For the sake of brevity, we say that a function $\psi$ on $\Omega \times \mathbb{T}$ has a deep zero if it satisfies

$$
s \in \mathbb{T} \mapsto\|\psi(\cdot, s)\|_{L^{p}(\Omega)} \in L^{1}(\mathbb{T}) \quad \text { and } \quad \int_{\mathbb{T}} \log \|\psi(\cdot, s)\|_{L^{p}(\Omega)} \frac{d s}{\tau}=-\infty .
$$

Let $\alpha>0$; we say that a function $f$ on the tree $\mathfrak{X}$ has $\alpha$-exponential decay if there exists $C>0$ such that

$$
|f(x)| \leq C e^{-\alpha|x|} \quad \forall x \in \mathfrak{X} .
$$

We measure the decay rate of a function $f$ on $\mathfrak{X}$ via the exponential scale as above. The following proposition shows that our result cannot be sharpened.

Proposition 2.2. There exists a nontrivial function $f$ on the tree $\mathfrak{X}$, whose Helgason-Fourier transform has a deep zero and with $\frac{1}{2} \log q$-exponential decay.

Conversely, there exists a nontrivial function $g$ on the tree $\mathfrak{X}$ with arbitrary exponential decay and whose Helgason-Fourier transform does not have deep zeroes.

Proof. Let $f$ be the inverse transform of the function

$$
\mathcal{H} f(\omega, s)= \begin{cases}e^{-\frac{1}{s \log q}} & \text { if } s>0, \\ 0 & \text { otherwise }\end{cases}
$$

Then $\mathcal{H} f$ has a deep zero on $\mathbb{T}$.

Moreover, since $\int_{\Omega} P^{\frac{1}{2}+i s}(x, \omega) d \sigma(\omega)=\phi_{s}(x)$, the spherical function [ 5 , Proposition 2.4] such that

$$
\phi_{s}(x)=\mathbf{c}(s) q^{(i s-1 / 2)|x|}+\mathbf{c}(-s) q^{(-i s-1 / 2)|x|} \quad \forall x \in \mathfrak{X},
$$


we have

$$
\begin{aligned}
f(x) & =\frac{q}{2(q+1)} \int_{0}^{\tau / 2} e^{-1 / s \log q} \phi_{-s}(x)|\mathbf{c}(s)|^{-2} \frac{d s}{\tau} \\
& =\frac{q}{2(q+1)} \int_{0}^{\tau / 2} e^{-1 / s \log q}\left(\mathbf{c}(s) q^{(i s-1 / 2)|x|}+\mathbf{c}(-s) q^{(-i s-1 / 2)|x|}\right)|\mathbf{c}(s)|^{-2} \frac{d s}{\tau} \\
& =\frac{q e^{-\frac{\log q}{2}|x|}}{2(q+1)} \int_{0}^{\tau / 2} e^{-1 / s \log q}\left(\mathbf{c}(-s)^{-1} q^{i s|x|}+\mathbf{c}(s)^{-1} q^{-i s|x|}\right) \frac{d s}{\tau} .
\end{aligned}
$$

In the last line we have used the well-known property $\mathbf{c}(-s)=\overline{\mathbf{c}(s)}$, which can be easily derived from the explicit formula of the $\mathbf{c}$-function for real $s$. Since the poles of $\mathbf{c}^{-1}$ are on $\{z \in \mathbb{C}: \operatorname{Im} z=1 / 2\}$, the last integral is clearly bounded uniformly in $x$ and one obtains that $f$ has $\frac{1}{2} \log q$-exponential decay.

Conversely, let $h_{t}$ be the heat kernel, i.e., the radial $t$-analytic solution of the equation

$$
\partial_{t} h_{t}=-L h_{t} \quad \text { and } \quad h_{0}=\delta_{o} .
$$

Its Helgason-Fourier transform is the function on $\Omega \times \mathbb{R}$ given by

$$
(\omega, s) \mapsto \exp [-t(1-\eta(s))]
$$

where, as before,

$$
\eta(z)=\frac{2 q^{1 / 2}}{q+1} \cos (z \log q) \quad \forall z \in \mathbb{C} .
$$

Hence it does not have deep zeroes. On the other hand, in [4, Proposition 2.5 (iii)] the following pointwise estimate is proved:

$$
h_{t}(x) \leq \frac{q^{1 / 2}(q+1)^{3}}{(q-1)^{3}} \frac{e^{-\frac{\left(q^{1 / 2}-1\right)^{2}}{q+1} t}}{t}\left(1+\frac{q-1}{q+1}|x|\right) q^{-|x| / 2} h_{t \eta(0)}^{\mathbb{Z}}(|x|+1),
$$

where $h_{t}^{\mathbb{Z}}$ denotes the heat kernel on $\mathbb{Z}$. In the same paper [4, Theorem 2.3] the authors established that

$$
h_{t \eta(0)}^{\mathbb{Z}}(|x|+1) \leq C \frac{\exp \left[-t \eta(0)+(|x|+1) \Phi\left(\frac{t \eta(0)}{|x|+1}\right)\right]}{\left(1+(|x|+1)^{2}+(t \eta(0))^{2}\right)^{1 / 4}},
$$

where $\Phi$ is the (increasing) function on $\mathbb{R}^{+}$defined by

$$
\Phi(r)=\sqrt{1+r^{2}}+\log \left(\frac{r}{1+\sqrt{1+r^{2}}}\right) \quad \forall r \in \mathbb{R}^{+} .
$$

Let $t$ be a fixed positive number. From these estimates one easily proves the decay $e^{-\alpha|x|}$ for $g=h_{t}$ for any $\alpha>\log q / 2$.

\section{REFERENCES}

1. F. Astengo, M. Cowling, B. Di Blasio, and M. Sundari, Hardy's Uncertainty Principle on certain Lie groups, J. London Math. Soc. 62 (2000), 461-472. MR 2002b:22018

2. W. Betori and M. Pagliacci, The Radon transform on trees, Boll. Un. Mat. Ital. 5-B (1986), 267-277. MR 87h:05073

3. M. G. Cowling, S. Meda and A. G. Setti, An overview of harmonic analysis on the group of isometries of a homogeneous tree, Exposition. Math. 16 (1998), 385-423. MR 2000i:43005

4. M. G. Cowling, S. Meda and A. G. Setti, Estimates for functions of the Laplace operator on homogeneous trees, Trans. Amer. Math. Soc. 352 (2000), 4271-4293. MR 2000m:43005] 
5. A. Figà-Talamanca and C. Nebbia, Harmonic Analysis and Representation Theory for Groups Acting on Homogeneous Trees, London Math. Soc. Lecture Notes Series, 162, Cambridge Univ. Press, Cambridge, 1991. MR 93f:22004

6. A. Figà-Talamanca and M. Picardello, Harmonic Analysis on Free Groups, Dekker, New York, 1983. MR 85j:43001

7. G. B. Folland and A. Sitaram, The uncertainty principle: a mathematical survey, J. Fourier Anal. Appl. 3 (1997), 207-238. MR 98f:42006

8. E. K. Narayanan and S. K. Ray, $L^{p}$ versions of Hardy's theorem on semisimple groups, Proc. Amer. Math. Soc. 130 (2002), 1859-1866. MR 2003a:22009

9. V. Havin and B. Jöricke, The Uncertainty Principle in Harmonic Analysis, Ergebnisse der Mathematik und ihrer Grenzgebiete, 3. Folge, Band 28, Springer-Verlag, Berlin, 1994. MR 96c:42001

10. S. Thangavelu, Hardy's Theorem for the Helgason Fourier transform on noncompact rank one symmetric spaces, preprint.

Dipartimento di Matematica, Università di Genova, 16146 Genova, Italia

E-mail address: astengo@dima.unige.it 